\title{
Avaliação de genótipos de Mentha arvensis, Mentha x piperita e Mentha spp. para a produção de mentol
}

\author{
Cícero Deschamps ${ }^{1}$; Rodrigo Monteiro'; Marília P Machado ${ }^{1}$; Agnes de P Scheer²; Lílian Cocco²; Carlos \\ Yamamoto $^{2}$ \\ 'UFPR, Dep ${ }^{\text {to }}$ Fitotecnia e Fitossanitarismo, C. Postal 19061, 81531-990 Curitiba-PR; cicero@ufpr.br; rodrimont@hotmail.com; \\ ma_rilia10@hotmail.com; ${ }^{2}$ UFPR/LACAUT, Dep ${ }^{\text {to }}$ Engenharia Química, Centro Politécnico, 81530-990 Curitiba-PR; agnesps@ufpr. \\ br; lilian.cocco@ufpr; ciyama@ufpr.br
}

\section{RESUMO}

O gênero Mentha possui 18 espécies e diversos quimiotipos com diferenças inter e intra-específicas. Apesar da grande variabilidade fitoquímica, o maior interesse econômico na produção de óleo essencial de menta deve-se à obtenção do constituinte mentol. Objetivou-se avaliar 19 genótipos de três espécies de Mentha para a produção de mentol. $\mathrm{O}$ experimento foi conduzido a campo com delineamento experimental em blocos casualizados, sendo avaliado o desenvolvimento vegetativo, a produtividade e a composição do óleo essencial em duas colheitas sucessivas. As mudas foram obtidas por estaquia a partir de plantas matrizes mantidas em estufa. O plantio foi realizado no mês de setembro e as colheitas em intervalos de 90 dias. Alguns genótipos, mesmo com elevada produção de biomassa, não apresentaram mentol na composição do óleo essencial. Destacou-se o 'Peppermint' (Mentha x piperita), produzindo $34,37 \mathrm{~mL} /$ planta de mentol na segunda colheita. Os genótipos de Mentha arvensis, tradicionalmente mais cultivados em função de seu alto teor de mentol, apresentaram menor produção de biomassa e produtividade de óleo essencial e de mentol devido à alta incidência e severidade de ferrugem.

Palavras-chave: Lamiaceae, menta, óleo essencial.

\begin{abstract}
Evaluation of Mentha arvensis, Mentha $\mathbf{x}$ piperita and Mentha spp. genotypes for menthol production

The genus Mentha has 18 species and many chemotypes with inter and intra specific differences. In spite of its great phytochemical diversity, the main economic interest on mint essential oil production is to obtain the constituent menthol. We evaluated the menthol production of 19 genotypes of three Mentha species. The experiment was carried out at field conditions in a randomized block design comparing the vegetative development, essential oil productivity and composition in two harvests. The plants were obtained by stem cuttings from adult plants maintained at greenhouse conditions. The plants were transferred to field at September and harvested in 90-day intervals. Some genotypes, even with high biomass production did not present menthol in the essential oil composition. The highest menthol production was obtained from the genotype 'Peppermint' (Mentha x piperita) with $34.37 \mathrm{~mL} /$ plant of menthol at the second harvest. The Mentha arvensis genotypes, traditionally grown because of its high menthol yield, presented low biomass production and essential oil and menthol productivity because of the high incidence and severity of "mint rust".
\end{abstract}

Keywords: Lamiaceae, mint, essential oil.

(Recebido para publicação em 7 de outubro de 2011; aceito em 15 de março de 2013)

(Received on October 7, 2011; accepted on March 15, 2013)

\begin{abstract}
A s espécies do gênero Mentha, popularmente conhecidas no Brasil como hortelã, são usadas para fins medicinais como analgésico, estomacal e intestinal, estimulante das funções cardíacas, controle da azia, gastrite, cólicas e gases (Grisi et al., 2006). Também são usadas como condimentares na forma de aromatizantes alimentícios e tempero in natura. Conforme Watanabe et al. (2006), dentre as espécies mais populares se destacam a menta do levante (M. citrata), hortelã verde (M. spicata), poejo (M. pulegium), hortelã crespa ( $M$. crispa), hortelã pimenta (M. x piperita)
\end{abstract}

e menta japonesa ou hortelã doce $(M$. arvensis).

Por ser considerada uma cultura exigente em fertilidade do solo e água, foi cultivada como desbravadora nas regiões do interior do Paraná e São Paulo, ocorrendo uma diminuição da área de cultivo com a diminuição das áreas desmatadas. Outros dois fatores que contribuíram para a diminuição da produção de menta foram o advento do mentol sintético e a suscetibilidade das plantas à doença conhecida como "ferrugem da menta", causada pelo fungo Puccinia menthae (Watanabe et al., 2006).

O principal produto das plantas do gênero Mentha é o óleo essencial que é produzido e armazenado em tricomas glandulares peltados, os quais estão presentes principalmente em folhas e flores e em menores densidades nos caules. Em algumas espécies o principal constituinte é o mentol, que possui grande aplicação nas indústrias farmacêutica, alimentícia e de cosméticos, movimentando aproximadamente US\$ 276 milhões de dólares ao ano com produção de 22,2 mil toneladas e produção estimada para 2010 de 30 mil toneladas 
(Sanganeria, 2005). Apesar do mercado promissor e rentável, a produção de menta no Brasil ainda se destina em sua maior parte para a produção de matéria seca para chás, sendo a grande maioria do óleo essencial de menta importado pela indústria nacional de países como China, Índia e Estados Unidos. No período de 2011 a julho de 2012, as importações do óleo essencial de mentas contabilizaram US\$32.321 mil relativos a $1.163 \mathrm{t}$, contra uma exportação de 42 $\mathrm{t}$ (Aliceweb, 2012).

As espécies de Mentha diferem entre si não somente morfologicamente, mas também em relação à produção e composição do óleo essencial (Silva et al., 2006). Segundo Grisi et al. (2006), a seleção entre espécies e dentro de uma mesma espécie, visando o aumento da produção de óleo essencial é uma ferramenta importante para os produtores de menta. Dentro dos processos de caracterização agronômica e melhoramento da espécie, devem ser considerados os fatores abióticos que afetam a produção e qualidade de óleo essencial.

O presente trabalho teve como objetivo comparar o desenvolvimento vegetativo e produção de óleo essencial e de mentol de Mentha arvensis, M. x piperita e $M$. spp.

\section{MATERIAL E MÉTODOS}

O experimento foi conduzido na zona rural do município de Campo

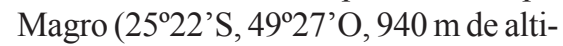
tude), Região Metropolitana de Curitiba, onde o clima é considerado subtropical úmido meso-térmico. $\mathrm{O}$ solo da área experimental é classificado como cambissolo háplico (Embrapa, 1999). Para a análise das características químicas procedeu-se a amostragem do solo, na profundidade de $0-20 \mathrm{~cm}$, conforme Comissão de Química e Fertilidade do Solo de RS e SC (2004). A amostra foi analisada segundo metodologia de Pavan et al. (1992) e apresentou: $\mathrm{pH}$ $\mathrm{CaCl}_{2}=4,90 ; \mathrm{Al}^{3+}=0,10 \mathrm{cmol}_{\mathrm{c}} \mathrm{dm}^{-3} ; \mathrm{H}^{+}$ $+\mathrm{Al}^{+3}=11,38 \mathrm{cmol}^{-3} ; \mathrm{Ca}^{\mathrm{c}}=3,70$ $\mathrm{cmol}_{\mathrm{c}} \mathrm{dm}^{-3} ; \mathrm{Mg}^{+2}=1,70 \mathrm{cmol}_{\mathrm{c}} \mathrm{dm}^{-3} ; \mathrm{K}^{+}=$ $0,18 \mathrm{cmol} \mathrm{dm}^{-3} ; \mathrm{P}=9,10 \mathrm{mg} \mathrm{dm}^{-3} ; \mathrm{C}=$ $19,0 \mathrm{~g} \mathrm{dm}^{-3}$; saturação de bases de $49 \%$ e teor de argila de $65 \mathrm{~g} \mathrm{~kg}^{-1}$. A correção da fertilidade e da acidez da área foi feita com base na recomendação de adubação e calagem do IAC (1997) para a cultura da menta. Foram aplicados $20 \mathrm{~kg} \mathrm{ha}^{-1} \mathrm{de}$ $\mathrm{N}, 40 \mathrm{~kg} \mathrm{ha}^{-1}$ de $\mathrm{P}_{2} \mathrm{O}_{5}, 60 \mathrm{~kg} \mathrm{ha}^{-1}$ de $\mathrm{K}_{2} \mathrm{O}$ e 2,4 $\mathrm{t} \mathrm{ha}^{-1}$ de calcário tipo Filler (PRNT $100 \%$ ), para elevar o índice $V$ para $70 \%$. A adubação de cobertura foi realizada com $30 \mathrm{~kg} \mathrm{ha}^{-1}$ de $\mathrm{N}$ aos 30 dias após o plantio. A segunda adubação foi realizada com $30 \mathrm{~kg} \mathrm{ha}^{-1}$ de $\mathrm{N}$ e $30 \mathrm{~kg} \mathrm{ha}^{-1}$ de $\mathrm{K}_{2} \mathrm{O}$, misturados e aplicados em toda a área, 21 dias após a primeira colheita. Antes do plantio os fertilizantes (uréia, super triplo e cloreto de potássio) e o calcário (tipo filler) foram incorporados por operação de gradagem.

Os genótipos avaliados pertencem às espécies $M$. arvensis, $M$. x piperita e Mentha spp. (Tabela 1). As mudas foram produzidas por estaquia herbácea de caule apresentando um comprimento médio de $10 \mathrm{~cm}$, com um par de folhas cortadas pela metade. O plantio foi feito em substrato contendo $50 \%$ de areia e $50 \%$ de Plantmax ${ }^{\circledR}$ hortaliças, permanecendo cerca de 30 dias em bandejas de isopor com 288 células, mantidas em câmara de nebulização intermitente, controlada por temporizador com intervalo de rega fixo. A programação utilizada para a nebulização foi das 8 às $17 \mathrm{~h}$, com irrigação de $15 \mathrm{~s}$ a cada 15 min; das 17 às $23 \mathrm{~h}$, com irrigação de $15 \mathrm{~s}$ a cada uma hora e; das 23 às $8 \mathrm{~h}$, com irrigação de $15 \mathrm{~s}$ a cada $3 \mathrm{~h}$, por um período de 15 dias. O bico nebulizador empregado apresenta vazão de $48 \mathrm{~L} \mathrm{~h}^{-1}$. O sombrite da câmara de nebulização é feito por manta termo-refletora Aluminet $^{\circledR}\left(\right.$ Polysack $\left.^{\circledR}\right)$.

O experimento foi implantado em 13 e 14 de outubro de 2006 a campo, com espaçamento de $0,50 \mathrm{~m}$ entre linhas e $0,45 \mathrm{~m}$ entre plantas, em parcelas de 9,0 $\mathrm{m}^{2}$ com 40 plantas cada. De cada parcela experimental foram avaliadas seis plantas. O delineamento experimental foi em blocos casualizados com três repetições. Durante os seis meses de cultivo, a irrigação foi realizada em dias alternados e o controle de plantas daninhas com capinas semanais. Houve incidência de ferrugem da menta (Puccinia menthae) nos genótipos de $M$. arvensis, sendo realizado o tratamento fitossanitário preventivo com calda bordalesa no segundo ciclo de cultivo (rebrota). A calda bordalesa foi preparada no mesmo dia da aplicação $\left(0,1 \% \mathrm{Cu}_{2} \mathrm{SO}_{4}+0,1 \% \mathrm{CaO}\right)$, sendo aplicados duas vezes por semana a partir da rebrota da primeira colheita; utilizou-se um pulverizador manual costal de 20 litros.

As colheitas ocorreram 90 dias após o plantio (verão) e 90 dias após a primeira colheita (outono). Estas foram feitas manualmente a $10 \mathrm{~cm}$ do solo utilizando-se tesoura de poda e separando-se a parte aérea dos estolões. Apenas os genótipos que apresentaram produções de mentol acima de $0,3 \mathrm{~mL}$ planta $^{-1}$ foram avaliados na segunda colheita.

Para cada colheita, o desenvolvimento vegetativo foi avaliado pela massa seca de ramos, folhas e estolões, obtida pela secagem de três amostras de $100 \mathrm{~g}$, de cada genótipo, de massa fresca em estufa a $65^{\circ} \mathrm{C}$, até massa seca constante.

Para a extração do óleo essencial foi utilizada uma amostra de $100 \mathrm{~g}$ de massa fresca de folhas para cada repetição, submetidas ao processo de hidrodestilação em aparelho graduado de Clevenger durante duas horas. $\mathrm{O}$ teor de óleo essencial foi corrigido para base seca após secagem de amostras a $65^{\circ} \mathrm{C}$ em estufa com ventilação forçada até atingir massa constante.

A análise do teor de mentol nas amostras de óleo essencial foi realizada por cromatografia em fase gasosa acoplada à espectrometria de massas (CG/ EM). O cromatógrafo utilizado foi da marca Varian, modelo CP-3800, com detector FID (CG/FID); coluna capilar Chrompack de sílica fundida CP-SIL 8 CB com 0,25 mm de diâmetro interno, $30 \mathrm{~m}$ de comprimento e $0,25 \mu \mathrm{m}$ de filme líquido. As condições de análise foram: a) temperatura do injetor: $250^{\circ} \mathrm{C}$, split $1: 200$; b) quantidade de amostra injetada: $1,0 \mu \mathrm{L}$; c) pressão da coluna: 30,0 psi; d) gás de arraste: hélio a 1,0 $\mathrm{mL} \min ^{-1}$ na temperatura de $60^{\circ} \mathrm{C}$; e) gás de make up: ar sintético, nitrogênio e hidrogênio; f) temperatura do detector FID: $300^{\circ} \mathrm{C} ; \mathrm{g}$ ) programação da temperatura do forno: inicial $60^{\circ} \mathrm{C}$, elevação da temperatura a $90^{\circ} \mathrm{C}$ na razão de $3^{\circ} \mathrm{C}$ permanecendo por 5 minutos, elevação da temperatura a $140^{\circ} \mathrm{C}$ na razão de $3^{\circ} \mathrm{C}$, elevação da temperatura a $240^{\circ} \mathrm{C}$ 
na razão de $30^{\circ} \mathrm{C}$ permanecendo por 5 minutos; h) tempo total de análise/ desenvolvimento: 40 minutos.

As análises de variância (ANOVA) foram realizadas utilizando o programa MSTAT-C (Nissen, 1993). As variâncias dos tratamentos foram testadas quanto à homogeneidade pelo teste de Bartlett e as médias dos tratamentos comparadas pelo teste de Tukey ao nível de 5\% de probabilidade.

\section{RESULTADOS E DISCUSSÃO}

Nas duas colheitas foram observadas diferenças significativas entre os genótipos para todas as variáveis analisadas. $\mathrm{Na}$ primeira colheita, a massa seca de ramos do genótipo 'Hortelã Botucatu' (Mentha spp.) foi de $3.420,49 \mathrm{~kg} \mathrm{ha}^{-1}$, sendo superior a todos os demais genótipos avaliados. Para a massa seca dos estolões os maiores valores entre os genótipos de Mentha spp. foram encontrados nos genótipos IAC 9, IAC 7 e IAC $5(1.328,73 ; 1.017,65$ e $758,97 \mathrm{~kg}$ $\mathrm{ha}^{-1}$, respectivamente), os quais não diferiram dos genótipos Menta do Uruguai, Hortelã 560, Peppermint e Chocolate mint, todos pertencentes à espécie $M$. x piperita. Os genótipos de $M$. arvensis foram significativamente inferiores para esta variável (Tabela 2). Os estolões podem ter importante função como estruturas de propagação, pois segundo Santos et al. (2012), a utilização de estolões para a propagação de $M$. canadensis é uma opção para a redução de custos na implantação da cultura.

Houve grande variação da massa seca de folhas, com produção de $3.535,23 \mathrm{~kg} \mathrm{ha}^{-1}$ no genótipo Menta do Uruguai (M. x piperita) até 169,83 $\mathrm{kg} \mathrm{ha}^{-1}$ no genótipo Hortelã-PR 1 ( $M$. arvensis). Os genótipos Ciudad Del Est (M. x piperita), Hortelã 1802, IAC 5, IAC 7, IAC 9 e Hortelã Botucatu (Mentha spp.) não diferiram significativamente do genótipo Menta do Uruguai. No genótipo Hortelã Botucatu foi obtida a maior massa seca total (6.992 $\left.\mathrm{kg} \mathrm{ha}^{-1}\right)$, não diferindo dos genótipos IAC 7, IAC 5 e Menta do Uruguai pertencentes à mesma espécie (Tabela 2).

As diferenças encontradas na massa seca de ramos, estolões e folhas entre os genótipos da espécie Mentha x piperita podem estar em parte relacionadas aos diferentes hábitos de crescimento, uma vez que as plantas de 'Ciudad Del Est' e 'Piperita Negra' apresentam crescimento ereto, e as plantas dos demais genótipos de $M$. x piperita apresentam hábito de crescimento rasteiro, com intensa ramificação estolonífera. Variações morfológicas e de desenvolvimento das plantas de menta foram avaliadas entre 25 genótipos, nas condições do Distrito Federal, demonstrando que o acúmulo de massa seca variou entre os diferentes genótipos estudados (Grisi et al., 2006). Nas condições experimentais do presente trabalho, é possível que alguns genótipos tenham tido seu potencial de produção reduzido em função das condições climáticas. Blank et al. (2005) relatam a importância de se conhecer o comportamento da espécie com relação aos efeitos climáticos da região de plantio, tratos culturais e os fatores bióticos que são responsáveis pelo desenvolvimento da planta.

A ocorrência da ferrugem, causada

por Puccinia menthae, nos genótipos de $M$. arvensis resultou em menor acúmulo de massa seca das plantas já que esta doença interfere na fisiologia da planta, reduzindo o desenvolvimento do sistema radicular, número de estolões, área foliar e massa seca da parte aérea (Edwards et al., 1999).

$\mathrm{Na}$ primeira colheita foram observadas diferenças significativas entre os 19 genótipos no teor e produtividade de óleo essencial e produtividade de mentol (Tabela 2).

Apesar da ocorrência de ferrugem, os genótipos de $M$. arvensis tiveram maiores teores de óleo essencial, quando comparados com os genótipos das espécies $M$. x piperita e Mentha spp. O maior teor de óleo essencial foi obtido com o genótipo Hortelã-PR 2 (17,0\%), não diferindo dos genótipos Hortelã 403 (13,49\%) e Hortelã-PR 1 (12,15\%). O menor teor de óleo essencial foi obtido no genótipo Menta do Uruguai $(1,02 \%)$ (Tabela 2).

A produtividade de óleo essencial foi superior no genótipo IAC 5

Tabela 1. Materiais genéticos de Mentha e suas respectivas procedências. (mint genetic materials and its respective origin). Campo Magro, UFPR, 2005.

\begin{tabular}{|c|c|c|}
\hline Nome cientifico & Nome comum & Procedência \\
\hline \multirow{4}{*}{ Mentha arvensis } & Hortelã 403 & CPQBA \\
\hline & Hortelã-PR 1 & Capitão Leônidas Marques \\
\hline & Hortelã-PR 2 & Capitão Leônidas Marques \\
\hline & Hortelã-PR 3 & Capitão Leônidas Marques \\
\hline \multirow{7}{*}{ Mentha $\mathrm{x}$ piperita } & Chocolate mint & Purdue University - EUA \\
\hline & Variegated mint & Purdue University - EUA \\
\hline & Peppermint & Purdue University - EUA \\
\hline & Ciudad Del Est & UnB \\
\hline & Piperita negra & UnB \\
\hline & Hortelã 560 & CPQBA \\
\hline & Hortelã 489 & CPQBA \\
\hline \multirow{8}{*}{ Mentha spp. } & Menta do Uruguai & Uruguai \\
\hline & Hortelã 1802 & São Paulo \\
\hline & IAC 5 & IAC \\
\hline & IAC 7 & IAC \\
\hline & IAC 8 & IAC \\
\hline & IAC 9 & IAC \\
\hline & Hortelã Botucatu & UNESP \\
\hline & UFC 5 & UFC \\
\hline
\end{tabular}

IAC: Instituto Agronômico de Campinas; UnB: Universidade de Brasília; CPQBA: Centro Pluridisciplinar de Pesquisas Químicas, Biológicas e Agrárias/UNICAMP; UFC: Universidade do Ceará; UNESP: Universidade do Estado de São Paulo. 
Tabela 2. Massa seca de ramos $\left(\mathrm{kg} \mathrm{ha}^{-1}\right)$, de estolões $\left(\mathrm{kg} \mathrm{ha}^{-1}\right)$, de folhas $\left(\mathrm{kg} \mathrm{ha}^{-1}\right)$, e total $\left(\mathrm{kg} \mathrm{ha}^{-1}\right)$, teor de óleo essencial (\%), produtividade de óleo essencial $\left(\mathrm{mL} \mathrm{planta}^{-1}\right)$ e produtividade de mentol $\left(\mathrm{mL} \mathrm{planta}^{-1}\right)$ de 19 genótipos de três espécies de Mentha, na primeira colheita (stem dry mass $\left(\mathrm{kg} \mathrm{ha}^{-1}\right)$, stolon dry mass $\left(\mathrm{kg} \mathrm{ha}^{-1}\right)$, leaf dry mass $\left(\mathrm{kg} \mathrm{ha}^{-1}\right)$, total dry mass $\left(\mathrm{kg} \mathrm{ha}^{-1}\right)$, essential oil yield (\%), essential oil productivity $\left(\mathrm{mL} \mathrm{plant}^{-1}\right)$ and menthol productivity $\left(\mathrm{mL} \mathrm{plant}^{-1}\right)$ of 19 genotypes of three mint species at the first harvest). Campo Magro, UFPR, 2005.

\begin{tabular}{|c|c|c|c|c|c|c|c|}
\hline Genótipo & $\begin{array}{c}\text { MS ramos } \\
\text { (kg/ha) }\end{array}$ & $\begin{array}{l}\text { MS estolões } \\
(\mathrm{kg} / \mathrm{ha})^{1}\end{array}$ & $\begin{array}{l}\text { MS folhas } \\
(\mathrm{kg} / \mathrm{ha})^{1}\end{array}$ & $\begin{array}{l}\text { MS total } \\
(\mathrm{kg} / \mathrm{ha})^{1}\end{array}$ & $\begin{array}{l}\text { Teor de OE } \\
(\%)^{1}\end{array}$ & $\begin{array}{c}\text { Produtividade } \\
\text { de OE } \\
\text { (mL/planta) }^{1}\end{array}$ & $\begin{array}{c}\text { Produtividade } \\
\text { de mentol } \\
(\mathrm{mL} / \text { planta })^{2}\end{array}$ \\
\hline Hortelã 403 & $892,1 \mathrm{e}-\mathrm{g}$ & $327,2 \mathrm{ef}$ & $251,9 \mathrm{hi}$ & $1471,0 \mathrm{ij}$ & $13,5 \mathrm{ab}$ & $15,1 d-g$ & $10,0 \mathrm{ab}$ \\
\hline Hortelã-PR 1 & $568,7 \mathrm{~g}$ & $210,4 \mathrm{fg}$ & $169,8 \mathrm{i}$ & $948,9 j$ & $12,2 \mathrm{ab}$ & $9,2 \mathrm{~g}$ & $6,1 \mathrm{bc}$ \\
\hline Hortelã-PR 2 & $1317,7 \mathrm{c}-\mathrm{g}$ & $93,7 \mathrm{~h}$ & $400,1 \mathrm{~h}$ & $1811,0 \mathrm{~g}-\mathrm{j}$ & $17,1 \mathrm{a}$ & $29,7 \mathrm{a}-\mathrm{c}$ & $19,1 \mathrm{a}$ \\
\hline Hortelã-PR 3 & $1076,0 \mathrm{e}-\mathrm{g}$ & $333,1 d-f$ & $382,6 \mathrm{~h}$ & $1792,0 \mathrm{~h}-\mathrm{j}$ & $9,3 \mathrm{bc}$ & $16,0 \mathrm{~d}-\mathrm{g}$ & $9,9 \mathrm{ab}$ \\
\hline Chocolate mint & $774,4 \mathrm{fg}$ & $1064,8 \mathrm{ab}$ & $892,6 \mathrm{fg}$ & $2732,0 \mathrm{~d}-\mathrm{i}$ & $2,7 \mathrm{e}-\mathrm{g}$ & $10,8 \mathrm{fg}$ & $3,6 b-d$ \\
\hline Variegated peppermint & $510,0 \mathrm{~g}$ & $450,3 c-e$ & $1123,6 \mathrm{e}-\mathrm{g}$ & $2084,0 e-j$ & $2,8 \mathrm{e}-\mathrm{g}$ & $14,4 \mathrm{~d}-\mathrm{g}$ & $0,0 \mathrm{e}$ \\
\hline Peppermint & $1117,9 \mathrm{~d}-\mathrm{g}$ & $778,4 a-c$ & $1500,7 \mathrm{c}-\mathrm{f}$ & $3397,0 \mathrm{~d}-\mathrm{g}$ & $2,1 \mathrm{f}-\mathrm{h}$ & $14,1 \mathrm{~d}-\mathrm{g}$ & $5,2 \mathrm{bc}$ \\
\hline Ciudad Del Est & $1354,0 \mathrm{c}-\mathrm{g}$ & $85,8 \mathrm{~h}$ & $2042,4 a-d$ & $3482,0 \mathrm{~d}-\mathrm{f}$ & $3,8 \mathrm{de}$ & $34,1 \mathrm{ab}$ & $0,0 \mathrm{e}$ \\
\hline Piperita negra & $2135,2 b c$ & $140,9 \mathrm{gh}$ & $1622,3 \mathrm{c}-\mathrm{e}$ & $3898,0 \mathrm{~cd}$ & $1,8 \mathrm{gh}$ & $13,1 \mathrm{e}-\mathrm{g}$ & $0,0 \mathrm{e}$ \\
\hline Hortelã 560 & $1020,6 \mathrm{e}-\mathrm{g}$ & $774,2 \mathrm{a}-\mathrm{c}$ & $1908,5 b-e$ & $3788,0 \mathrm{~cd}$ & $2,8 \mathrm{e}-\mathrm{g}$ & $23,8 b-d$ & $8,5 b$ \\
\hline Hortelã 489 & $1553,9 \mathrm{c}-\mathrm{f}$ & $635,8 b-d$ & $1422,4 d-f$ & $3612,0 \mathrm{de}$ & $6,0 \mathrm{~cd}$ & $37,0 \mathrm{ab}$ & $0,3 \mathrm{de}$ \\
\hline Menta do Uruguai & $1947,45 b-d$ & $1036,5 \mathrm{ab}$ & $3535,2 \mathrm{a}$ & $6519,0 \mathrm{ab}$ & $1,0 \mathrm{i}$ & $14,8 \mathrm{~d}-\mathrm{g}$ & $0,0 \mathrm{e}$ \\
\hline Hortelã 1802 & $1580,58 \mathrm{c}-\mathrm{f}$ & $390,1 d-f$ & $2221,5 \mathrm{a}-\mathrm{d}$ & $4192,0 \mathrm{~cd}$ & $3,1 \mathrm{ef}$ & $30,9 a-c$ & $0,0 \mathrm{e}$ \\
\hline IAC 5 & $2534,0 \mathrm{~b}$ & $759,0 \mathrm{a}-\mathrm{c}$ & $3147,8 \mathrm{ab}$ & $6441,0 \mathrm{ab}$ & $3,3 \mathrm{ef}$ & $45,9 \mathrm{a}$ & $0,0 \mathrm{e}$ \\
\hline IAC 7 & $1738,0 \mathrm{~b}-\mathrm{e}$ & $1017,7 \mathrm{ab}$ & $3177,5 \mathrm{ab}$ & $5933,0 \mathrm{ab}$ & $2,7 \mathrm{e}-\mathrm{g}$ & $38,5 \mathrm{ab}$ & $0,0 \mathrm{e}$ \\
\hline IAC 8 & $1153,2 \mathrm{~d}-\mathrm{g}$ & $400,3 d-f$ & $1630,1 \mathrm{c}-\mathrm{e}$ & $3184,0 \mathrm{~d}-\mathrm{h}$ & $3,0 \mathrm{ef}$ & $21,8 \mathrm{~b}-\mathrm{e}$ & $0,0 \mathrm{e}$ \\
\hline IAC 9 & $1453,4 c-f$ & $1328,7 \mathrm{a}$ & $2592,8 \mathrm{a}-\mathrm{c}$ & $5375,0 \mathrm{bc}$ & $2,7 \mathrm{e}-\mathrm{g}$ & $30,8 \mathrm{a}-\mathrm{c}$ & $6,5 \mathrm{bc}$ \\
\hline Hortelã Botucatu & $3420,5 \mathrm{a}$ & $411,9 c-e$ & $3159,8 \mathrm{ab}$ & $6992,0 \mathrm{a}$ & $1,3 \mathrm{hi}$ & $18,6 \mathrm{c}-\mathrm{f}$ & $0,0 \mathrm{e}$ \\
\hline UFC 5 & $916,2 \mathrm{e}-\mathrm{g}$ & $239,0 \mathrm{e}-\mathrm{g}$ & $780,8 \mathrm{~g}$ & $1936,0 f-j$ & $1,5 \mathrm{hi}$ & $5,3 \mathrm{~h}$ & $1,8 \mathrm{c}-\mathrm{e}$ \\
\hline$\overline{\mathrm{CV}(\%)}$ & 19,48 & 3,49 & 2,41 & 13,99 & 4,79 & 8,04 & 25,91 \\
\hline
\end{tabular}

${ }^{1}$ Os dados originais foram transformados em $\log (\mathrm{x})$ para análise estatística (the original data were transformed to $\log (\mathrm{x})$ for statistical analysis); ${ }^{2}$ Os dados originais foram transformados em raiz de $(\mathrm{x}+1)$ para análise estatística (the original data were transformed to square root of $(x+1)$ for statistical analysis); Médias seguidas pela mesma letra na coluna não diferem estatisticamente entre si pelo teste de Tukey a $5 \%$ de probabilidade (means followed by the same letter do not differ by Tukey test at 5\% of probability).

(Mentha spp.), com média de 103,32 $\mathrm{L} \mathrm{ha}^{-1}$, porém sem diferença significativa com os genótipos IAC 9, IAC 7, Hortelã 1802, Hortelã 489, Ciudad Del Est e Hortelã-PR2 (Tabela 2). A produtividade de óleo essencial depende do teor e da produção de biomassa foliar; por isso genótipos com baixo teor de óleo essencial, como IAC 5, apresentaram altas produtividades devido à maior produção de biomassa. Comportamento semelhante foi observado em outros genótipos de Mentha spp. (Czepak, 1995; Garlet et al., 2007; Chagas et al., 2011).

$\mathrm{Na}$ análise cromatográfica dos óleos essenciais, foram identificadas espécies não produtoras de mentol, fato já observado anteriormente em menta (Deschamps et al., 2008). Os genótipos que apresentaram alta produtividade de óleo essencial, porém sem mentol em sua composição, não foram avaliados na segunda colheita. Segundo Grisi et al. (2006), as variações no teor de óleo essencial estão relacionadas às diferenças genéticas das plantas. É demonstrado nos estudos de biossíntese de óleos essenciais que as variações no teor e composição do óleo são função dos aspectos ambientais que interferem na expressão gênica das plantas (Marotti et al., 1994). As variações no teor de composição do óleo essencial de genótipos de Mentha, já foram demonstradas em outros estudos (Telci et al., 2004; Gracindo et al., 2006; Deschamps et al., 2008; Zaks et al., 2008).

O genótipo Hortelã-PR 2 de $M$. arvensis apresentou maior produtividade de mentol $\left(19,13 \mathrm{~mL}\right.$ planta $\left.^{-1}\right)$, porém sem diferença significativa com os genótipos 'Hortelã 403' $(10,04 \mathrm{~mL}$ planta $\left.^{-1}\right)$ e 'Hortelã-PR 3' $(9,9$ mL planta $^{-1}$ ) (Tabela 2). Esses resultados estão de acordo com Ozel \& Ozguven (2002) que também obtiveram maiores produtividades de mentol em M. arvensis.

A produção de massa seca de ramos na segunda colheita foi superior no genótipo Hortelã-PR 3 da espécie M. arvensis $\left(2.451,85 \mathrm{~kg} \mathrm{ha}^{-1}\right)$, não diferindo estatisticamente do genótipo Peppermint de M. x piperita $(2.185,19$ $\left.\mathrm{kg} \mathrm{ha}^{-1}\right)$. Os genótipos IAC 9 e UFC 5 da espécie Mentha spp., apresentaram menor acúmulo de massa seca de ramos, com 824,75 e $432,4 \mathrm{~kg} \mathrm{ha}^{-1}$, respectivamente (Tabela 3).

Os genótipos Peppermint e Hortelã 560 de $M$. x piperita apresentaram 
Tabela 3. Massa seca de ramos $\left(\mathrm{kg} \mathrm{ha}^{-1}\right)$, de folhas $\left(\mathrm{kg} \mathrm{ha}^{-1}\right)$, e total $\left(\mathrm{kg} \mathrm{ha}^{-1}\right)$, produtividade óleo essencial $\left(\mathrm{mL} \mathrm{planta}^{-1}\right)$ e produtividade de mentol ( $\left.\mathrm{mL} \mathrm{planta}^{-1}\right)$ de nove genótipos de três espécies de Mentha, na segunda colheita (stem dry mass $\left(\mathrm{kg} \mathrm{ha}^{-1}\right)$, leaf dry mass $\left(\mathrm{kg} \mathrm{ha}^{-1}\right)$, total dry mass $\left(\mathrm{kg} \mathrm{ha}^{-1}\right)$, essential oil productivity $\left(\mathrm{mL} \mathrm{plant}^{-1}\right)$ and menthol productivity $\left(\mathrm{mL} \mathrm{plant}^{-1}\right)$ of nine genotypes of three mint species at the second harvest). Campo Magro, UFPR, 2006.

\begin{tabular}{lccccc}
\hline Genótipo & $\begin{array}{c}\text { MS ramos } \\
\mathbf{( k g / h a )}\end{array}$ & $\begin{array}{c}\text { MS folhas } \\
\mathbf{( k g / h a )}\end{array}$ & $\begin{array}{c}\text { MS total } \\
\mathbf{( k g / h a )}\end{array}$ & $\begin{array}{c}\text { Produtividade OE } \\
\text { (mL/planta) }\end{array}$ & $\begin{array}{c}\text { Produtividade } \\
\text { de mentol } \\
\text { (mL/planta) }\end{array}$ \\
\hline Hortelã 403 & $1075,60 \mathrm{de}$ & $203,60 \mathrm{~d}$ & $1131,21 \mathrm{~b}$ & $19,26 \mathrm{de}$ & $12,84 \mathrm{~cd}$ \\
Hortelã-PR 1 & $1663,70 \mathrm{c}$ & $310,60 \mathrm{c}$ & $1827,13 \mathrm{ab}$ & $15,49 \mathrm{e}$ & $10,20 \mathrm{~d}$ \\
Hortelã-PR 2 & $1138,60 \mathrm{~d}$ & $219,95 \mathrm{~cd}$ & $1221,94 \mathrm{~b}$ & $23,54 \mathrm{~d}$ & $15,36 \mathrm{~cd}$ \\
Hortelã-PR 3 & $2451,85 \mathrm{a}$ & $495,16 \mathrm{~b}$ & $2606,11 \mathrm{ab}$ & $24,70 \mathrm{~d}$ & $15,24 \mathrm{~cd}$ \\
Peppermint & $2185,19 \mathrm{ab}$ & $1232,89 \mathrm{a}$ & $3082,22 \mathrm{a}$ & $93,73 \mathrm{a}$ & $34,37 \mathrm{a}$ \\
Chocolate mint & $1532,50 \mathrm{c}$ & $673,18 \mathrm{~b}$ & $2039,94 \mathrm{ab}$ & $52,03 \mathrm{bc}$ & $17,31 \mathrm{c}$ \\
Hortelã 560 & $2131,20 \mathrm{~b}$ & $1095,97 \mathrm{a}$ & $2739,94 \mathrm{ab}$ & $64,09 \mathrm{~b}$ & $24,34 \mathrm{~b}$ \\
IAC 9 & $824,75 \mathrm{e}$ & $723,37 \mathrm{~b}$ & $1446,73 \mathrm{ab}$ & $60,82 \mathrm{bc}$ & $12,84 \mathrm{~cd}$ \\
UFC 5 & $432,40 \mathrm{f}$ & $591,73 \mathrm{~b}$ & $1479,32 \mathrm{ab}$ & $43,83 \mathrm{c}$ & $15,07 \mathrm{~cd}$ \\
\hline CV (\%) & 6,91 & 2,19 & 10,57 & 3,06 & 13,32 \\
\hline
\end{tabular}

${ }^{1}$ Os dados originais foram transformados em $\log (\mathrm{x})$ para análise estatística (the original data were transformed to $\log (\mathrm{x})$ for statistical analysis); Médias seguidas pela mesma letra na coluna não diferem estatisticamente entre si pelo teste de Tukey a 5\% de probabilidade (means followed by the same letter do not differ by Tukey test at 5\% of probability).

maior produção de massa seca foliar na segunda colheita $(1.232,89$ e $1.095,97$ $\mathrm{kg} \mathrm{ha}^{-1}$, respectivamente). O genótipo Hortelã 403 de M. arvensis por sua vez foi inferior, aos demais genótipos avaliados, exceto ao genótipo Hortelã-PR 2, apresentado em média 203,60 $\mathrm{kg} \mathrm{ha}^{-1}$. Esses resultados podem ter refletido na produtividade de óleo essencial, uma vez que foi demonstrado anteriormente, com diferentes espécies de Mentha, que a produção de biomassa foliar é o fator determinante na produtividade de óleo essencial (Ram \& Kumar, 1997; Rao, 2002; Aflatuni, 2005; Monteiro et al., 2011).

A produção de massa seca total no genótipo Peppermint ( $M$. x piperita) $\left(3.082,22 \mathrm{~kg} \mathrm{ha}^{-1}\right)$ foi superior apenas em relação aos genótipos Hortelã 403 e Hortelã-PR 2 (M. arvensis), com massa seca total de $1.131,21 \mathrm{~kg} \mathrm{ha}^{-1} \mathrm{e} 1.221,94$ $\mathrm{kg} \mathrm{ha}^{-1}$, respectivamente (Tabela 3).

A redução do acúmulo de massa seca em alguns genótipos após a primeira colheita ocorreu em função do menor desenvolvimento das plantas pelo fim da estação do verão e início do outono. Estudos realizados por Clark \& Menary (1984) relatam diminuição na produção de massa seca de Mentha x piperita em colheitas de rebrotes, assim como Aflatuni (2005) e Ram \& Kumar (1997) em M. arvensis. Contudo, os genótipos de $M$. arvensis 'Hortelã-PR 1' e 'Hortelã- PR 3', apresentaram aumento na massa seca total quando comparada à primeira colheita (Tabelas 2 e 3). O genótipo Peppermint ( $M$. x piperita) foi o que apresentou maior produtividade de óleo essencial e de mentol na segunda colheita (Tabela 3).

Em contraste com os resultados obtidos por diversos autores, que encontraram menores valores de produtividade de óleo essencial e mentol a partir da segunda colheita (Deschamps et al., 2008; Aflatuni, 2005; Kofidis et al., 2004; Croteau et al., 2005), pode-se observar que os genótipos Hortelã 403, Hortelã-PR 1 e Hortelã-PR 3 de $M$. arvensis apresentaram produtividades superiores na segunda colheita (Tabelas 2 e 3 ). Esse aumento de produtividade de óleo essencial pode ser uma resposta ao controle da ferrugem nesses genótipos, com a aplicação preventiva de calda bordalesa. Já o genótipo Hortelã-PR 2 teve a sua produtividade reduzida em aproximadamente $20 \%$, na segunda colheita (Tabelas 2 e 3), provavelmente devido ao controle ineficiente da ferrugem para esse genótipo, já que a incidência da ferrugem na menta reduz a produção de óleo essencial (Edwards et al., 1999; May et al., 2008).
Foram observadas diferenças entre os genótipos em relação ao acúmulo de biomassa e produção de óleo essencial nas duas colheitas. Porém, algumas espécies apesar de apresentarem alta produção de biomassa e óleo essencial, não apresentam mentol na sua constituição, o que inviabiliza o cultivo para essa finalidade. Entre as espécies produtoras de mentol, alguns genótipos apresentaram baixo acúmulo de biomassa e produtividade de óleo essencial e mentol, sugerindo a necessidade de mais estudos para incremento da produção.

Os genótipos avaliados apresentam maior desenvolvimento vegetativo na primeira colheita (verão) em relação à segunda colheita (outono). Porém, para produtividade de óleo essencial e mentol, a maioria dos genótipos atingiu maiores valores na segunda colheita.

A partir dos resultados encontrados neste trabalho, conclui-se que os genótipos potenciais para a produção de mentol são Hortelã 403, Hortelã PR 1, Hortelã PR 2, Hortelã PR 3 ( $M$. arvensis), Peppermint, Chocolate mint, Hortelã 560 ( $M$. x piperita), IAC 9, UFC 5 (Mentha spp.), com produtividades de óleo essencial e de mentol variando de 34,22 a 104,67 mL planta $^{-1}$ e de 16,25 a $37,98 \mathrm{~L} \mathrm{ha}^{-1}$, respectivamente, após os dois primeiros cortes. 


\section{AGRADECIMENTOS}

Os autores agradecem ao Dr. Roberto Vieira e Dr. Dijalma Barbosa (Embrapa Recursos Geneticos) pelo fornecimento do material vegetal utilizado no trabalho e ao Conselho Nacional de Desenvolvimento Científico e Tecnológico $(\mathrm{CNPq})$ pela concessão de bolsa ao primeiro autor.

\section{REFERÊNCIAS}

AFLATUNI A. 2005. The yield and essential content of mint (Mentha ssp) in northern Ostrobothnia. Oulu: Universidade de Oulu. $50 \mathrm{p}$ (Tese mestrado).

ALICEWEB - Sistema de Análise das Informações de Comércio Exterior. 2012, 31 de outubro. Ministério do Desenvolvimento, Indústria e Comércio Exterior. Disponível em http:// aliceweb.desenvolvimento.gov.br/

BLANK AF; FONTES SM; OLIVEIRA AS; MENDONÇA MC; SILVA-MANN R; ARRIGONI-BLANK MF. 2005. Produção de mudas, altura e intervalo de corte em melissa. Horticultura Brasileira 23: 780-784.

CHAGAS JH; PINTO JEBP; BERTOLUCCI SKV; SANTOS FM. 2011. Produção de biomassa e teor de óleo essencial em função da idade e época de colheita em plantas de hortelã-japonesa. Acta Scientiarum Agronomy 33: 327-334.

CLARK RJ; MENARY RC. 1984. The effect of two harvest per year on the yield and the composition of Tasmanian peppermint oil (Mentha piperita L.). Journal of the Science of Food and Agriculture 35: 1191-1195.

COMISSÃO DE QUÍMICA E FERTILIDADE DO SOLO. 2004: Manual de Adubação e calagem para os Estados do Rio Grande do Sul e Santa Catarina. 2004. 1 ed. SBCS. Núcleo Regional Sul, 400p.

CROTEAU R; DAVIS EM; RINGER KL; WILDUNG MR. 2005. (-)- Menthol biosynthesis and molecular genetics. Naturwissenschaften 92: 562-577.

CZEPAK MP. 1995. Produção de óleo e mentol cristalizável em oito frequências de colheita da menta (Mentha arvensis L.). Piracicaba: Universidade de São Paulo. 81p. (Dissertação mestrado).

DESCHAMPS C; ZANATTA JL; BIZZO HR;
OLIVEIRA MC; ROSWALKA LC. 2008. Avaliação sazonal do teor de óleo essencial em espécies de menta. Ciência e Agrotecnologia 32: 725-730.

EDWARDS J; PARBERY DG; TAYLOR PA; HALLORAN GM. 1999. Effects of Puccinia menthae on growth and yield of Todd's Mitcham peppermint. Australian Journal of Agricultural Research 50: 1273-1278.

EMPRESA BRASILEIRA DE PESQUISA AGROPECUÁRIA (EMBRAPA).1999. Sistema Brasileiro de Classificação de solos. Embrapa Produção de Informação.

GARLET TMB; SANTOS OS; MEDEIROS SLP; MANFRON PA; GARCIA DC; SINCHAK SS. 2007. Crescimento e teor de óleo essencial de mentas com diferentes concentrações de potássio na solução nutritiva. Horticultura Brasileira 25: 230-237.

GRACINDO LAMB; GRISI MCM; SILVA DB; ALVES RBN; BIZZO HR; VIEIRA RF. 2006. Chemical characterization of mint (Mentha spp.) germplasm at Federal District, Brazil. Revista Brasileira de Plantas Medicinais 8: 5-9.

GRISI MCM; SILVA DB; ALVES RBN; GRACINDO LAMB; VIEIRA RF. 2006. Avaliação de genótipos de Menta (Mentha spp) nas condições do Distrito Federal, Brasil. Revista Brasileira de Plantas Medicinais 8: 33-39.

INSTITUTO AGRONÔMICO DE CAMPINAS (IAC). 1997. Recomendação de adubação e calagem para o Estado de São Paulo. Campinas: Instituto Agronômico, Fundação IAC.

KOFIDIS G; BOSABALIDIS A; KOKKINI S. 2004. Seasonal variation of essential oils in a linalool-rich chemotype of Mentha spicata grown wild in Greece. Journal of Essential oil research 16: 469-472.

MAROTTI M; PICCAGLIA R; GIOVANELLIE; DEANS SG; EAGLESHAM E. 1994. Effects of planting time and mineral fertilization on peppermint (Mentha x piperita L.) essential oil composition and its biological activity. Journal of Flavour and Fragrance 9: 125-129.

MAY A; TANAKA MAS; SILVA EHFM; MORAES ARA. 2008. Ferrugem da Mentha citrata no estado de São Paulo. Campinas: Instituto Agronômico. 8p.

MONTEIRO R; DESCHAMPS C; BIASI LA; BIZZO H. 2011. Desenvolvimento vegetativo de Mentha campestris Schur e produção de mentol em diferentes espaçamentos de plantio e épocas de colheita. Revista Brasileira de Plantas Medicinais 13: 401-407.
NISSEN, O. 1993. MSTAT-C. A microcomputer for design, management and analysis of Agronomic research experiments. Version 2.11. East Lansing, Michigan State University. $300 \mathrm{p}$.

OZELA; OZGUVEN M. 2002. Effect of different planting times on essential oil components of different mint (Mentha spp.) varieties. Turkish Journal of Agriculture and Forestry 26: 289-294.

PAVAN MA; BLOCH MF; ZEMPULSKI HC; MIYAZAWA M; ZOCOLER DC. 1992. Manual de análise química de solo e controle de qualidade. IAPAR. Circular 76.

RAM M; KUMAR S. 1997. Yield improvement in the regenerated and transplanted mint Mentha arvensis by recycling the organic wastes and manures. Bioresource Technology 59: 141-149.

RAO BRR. 2002. Biomass yield, essential oil yield and essential oil composition of rose-scented geranium (Pelargonium species) as influenced by row spacings and intercropping with cornmint (Mentha arvensis L. f. piperascens Malinv. Ex Holmes). Industrial Crops and Products 16: 133-144.

SANGANERIA S. 2005. Vibrant India. Opportunities for the flavor and fragrance industry. Perfumer \& Flavorist 30: 24-34.

SANTOS VMCS; SCHNEIDER TR; BIZZO HR; DESCHAMPS C. 2012. Alternativa de propagação na produção de óleo essencial de Mentha canadensis L. no Litoral Norte Catarinense. Revista Brasileira de Plantas Medicinais 14: 97-102.

SILVA DB; VIEIRA RF; ALVES RBN; MENDES RA; CARDOSO LD; QUEIROZ L; SANTOS IRI. 2006. Mint (Mentha spp.) germplasm conservation in Brazil. Revista Brasileira de Plantas Medicinais 8: 27-31.

TELCI I; SAHBAZ N; YILMAZ G; TUGAY ME. 2004. Agronomical and chemical characterization of spermint (Mentha spicata L.) originating in Turkey. Economic Botany 4: 721-728.

WATANABE CH; NOSSE TM; GARCIA CA; PINHEIRO POVH N. 2006. Extração do óleo essencial de menta (Mentha arvensis L.) por destilação por arraste a vapor e extração com etanol. Revista Brasileira de Plantas Medicinais 4: 76-86.

ZAKS A; DAVIDOVICH-RIKANATI R; INBAR M; LEWINSOHN E. 2008. Biosynthesis of linalyl acetate and other terpenes in lemon mint (Mentha aquatica var. citrata, Lamiaceae) glandular trichomes. Israel Journal of Plant Sciences 56: 233-244. 